$>$ Des travaux récents réalisés in vitro montrent que l'appauvrissement du milieu de culture en oxygène (hypoxie) active la réplication du parvovirus humain B19, des virus du sarcome de Kaposi et de l'immunodéficience humaine, ainsi que l'expression de protéines virales oncogènes. Les mécanismes de cette régulation impliquent le plus souvent le facteur cellulaire majeur de réponse à l'hypoxie, HIF-l (hypoxia inducible factor-1). Le dérèglement de ce facteur de transcription participe également au pouvoir oncogène de certains de ces virus. <

\title{
Interaction des virus avec la voie cellulaire de réponse à l'hypoxie
}

\author{
Sylvie Pillet, Nathalie Le Guyader
}

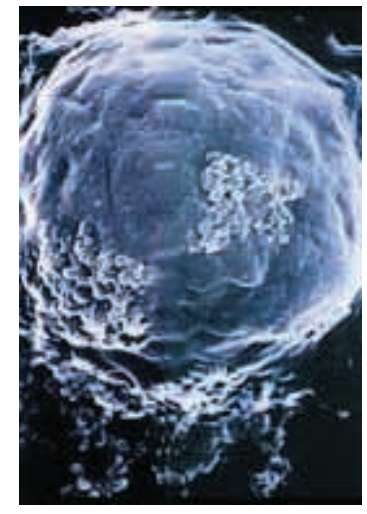

Réponse cellulaire à l'hypoxie
S. Pillet : Laboratoire de bactériologie-virologie, Hôpital Nord et Faculté de médecine Jacques Lisfranc, CHU de Saint-غ́tienne, 27, boulevard Pasteur, 42100 Saint-Étienne, France. N. Le Guyader : Service de pharmacie, Hôpital Armand Trousseau, 26, avenue du Docteur Arnold Netter, 75012 Paris, France. sylvie.pillet@univ-st-etienne.fr
La mesure de la pression partielle en oxygène $\left(\mathrm{pO}_{2}\right)$ dans les tissus humains montre qu'elle est très largement inférieure à celle de l'air que nous respirons. En effet, si la $\mathrm{pO}_{2}$ est de $15 \%$ au niveau des alvéoles pulmonaires, elle n'est que de $4 \%$ dans le foie, de $3 \%$ au niveau de la moelle osseuse et de $2 \%$ au niveau des cellules tubulaires rénales $[1,2]$. Ces conditions d'hypoxie relative dans l'organisme correspondent en fait à un état de «normoxie » physiologique puisqu'elles sont compatibles avec le fonctionnement normal des cel- $(\rightarrow) \mathrm{m} / \mathrm{s}$ 2003, $n^{\circ} 11$, p. 1120 lules et des tissus in vivo. Ainsi, le développement fœto-placentaire [3] $(\rightarrow)$, la formation du cartilage par les chondrocytes [4], ou encore la différenciation et la maturation des précurseurs hématopoïétiques [5] ne se réalisent correctement qu'à faible concentration d'oxygène. Notons que la $\mathrm{pO}_{2}$ des étuves dans lesquelles sont incubées les cellules utilisées pour la réplication des virus in vitro est de $20 \%$ et donc bien loin de respecter ces conditions physiologiques.

L'hypoxie tissulaire au sens strict se définit comme une concentration en oxygène inférieure aux valeurs physiologiques et qui ne permet pas de satisfaire aux besoins des tissus [1]. Ces conditions sont décrites, par exemple, au cours d'ischémies lors des angéites [6] ou au sein des tumeurs solides [7].

Article reçu le 19 novembre 2004 et accepté le 26 janvier 2005

Les mécanismes impliqués dans la réponse cellulaire à l'hypoxie ont fait l'objet d'un article récent $(\rightarrow)$ et ne $(\rightarrow) \mathrm{m} / \mathrm{s}$ $2002, n^{\circ} 1$, p. 70 seront rappelés ici que très brièvement (Figure 1) [8]. Le facteur majeur de réponse à l'hypoxie est nommé HIF-1 (hypoxia inducible factor-1). Il s'agit d'un hétérodimère constitué de deux protéines de la famille bHLH-PAS : HIF$1 \beta$ ou ARNT (aryl hydrocarbon receptor nuclear translocator) et HIF-l $\alpha(\rightarrow)$.

C'est la régulation de la stabilité et de l'activité transcriptionnelle de la sousunité $\alpha$ par la concentration d' $\mathrm{O}_{2}$ intracellulaire qui module l'activité du fac-

$(\rightarrow) \mathrm{m} / \mathrm{s}$ $1999, n^{\circ} 12$, p. 1379

$\rightarrow) \mathrm{m} / \mathrm{s}$ 2002, $n^{\circ} 1$, p. 70 teur HIF-1 $(\rightarrow)$.

Dès que la tension partielle en oxygène est inférieure à $6 \%$, la protéine HIF- $1 \alpha$ est stabilisée, migre dans le noyau cellulaire et s'associe à son partenaire HIF-1 $\beta$; le dimère ainsi formé recrute de nombreux cofacteurs, en particulier $\mathrm{CBP} / \mathrm{p} 300$. Le complexe transcriptionnel actif va alors se fixer sur un motif spécifique présent dans les régions régulatrices des gènes cibles et nommé HBS (HIF-binding site). Ce motif a pour séquence consensus ${ }^{5}$ 'RCGTG ${ }^{3}$ ' où $R$ est une purine. Cette séquence est retrouvée dans les régions régulatrices de nombreux 
gènes cellulaires dont l'expression est contrôlée par la tension en oxygène. Elle est ainsi présente dans la région 3' régulatrice (enhancer) du gène codant pour l'érythropoïétine ( $\varepsilon p o)$ et participe au contrôle de la synthèse de l'Epo par les cellules péritubulaires rénales en réponse à une diminution du taux de l'hémoglobine. Cette séquence est également retrouvée dans le promoteur du gène codant pour le VEGF (vascular endothelial growth factor) et son activation, en hypoxie, participe à la néo-angiogenèse tumorale $\left(\mathrm{pO}_{2}\right.$ intra-tumorale inférieure à $1 \%$ ).

\section{Virus et réponse cellulaire à l'hypoxie}

Des études récentes montrent que des virus peuvent interférer avec la voie cellulaire de réponse à l'hypoxie, soit en perturbant la régulation de l'expression d'HIF-l $\alpha$ dans le cas des virus oncogènes, soit en utilisant le facteur de transcription HIF-l pour augmenter l'expression de leurs gènes (Tableau I).

\section{Protéines virales oncogènes et régulation d'HIF- $1 \alpha$}

Les protéines virales oncogènes, en activant notamment la voie des MAPkinases, sont capables de stabiliser et d'activer HIF-l $\alpha$. Par ce mécanisme, l'oncoprotéine $H B x$ du virus de l'hépatite $B$ participe à la néo-angiogenèse de I'hépatocarcinome en favorisant la production de VEGF $[9,10]$ (Figure 2A). De plus, $H B x$ interagit directement avec HIF-l $\alpha$, empêchant ainsi sa dégradation et favorisant son interaction avec ses partenaires transcriptionnels [11]. Notons que la synthèse de HBx est également augmentée dans des conditions d'hypoxie in vitro (Tableau I) [12] et que $\mathrm{HBx}$ est capable de stimuler l'activité transcriptionnelle de son propre promoteur in vitro ; cette boucle d'autorégulation amplifie encore la production de facteurs pro-angiogéniques.

De même, la protéine membranaire LMPI (latent membrane protein 1) codée par le virus d'Epstein-Barr est susceptible d'activer de nombreuses voies de transduction, en particulier celles des MAPkinases et des dérivés réactifs de l'oxygène, et ainsi d'augmenter l'expression de HIF-l $\alpha$ in vitro (Figure 2B) [13]. condition de normoxie.
Ce phénomène, reproduit dans un modèle in vitro, pourrait expliquer l'augmentation de l'expression de HIF- $1 \alpha$ et de VEGF observée sur des coupes de cancer du nasopharynx, dans lequel la protéine LMPl est surexprimée [14].

Le génome du virus herpès 8 (HHV8) code pour des protéines qui possèdent de fortes homologies fonctionnelles avec des médiateurs cellulaires. Ainsi, la protéine vGPCR (viral G protein-coupled receptor) présente une homologie importante avec le récepteur cellulaire de l'interleukine-8, chimiokine qui possède des propriétés pro-angiogéniques. Le domaine intra-cytoplasmique de

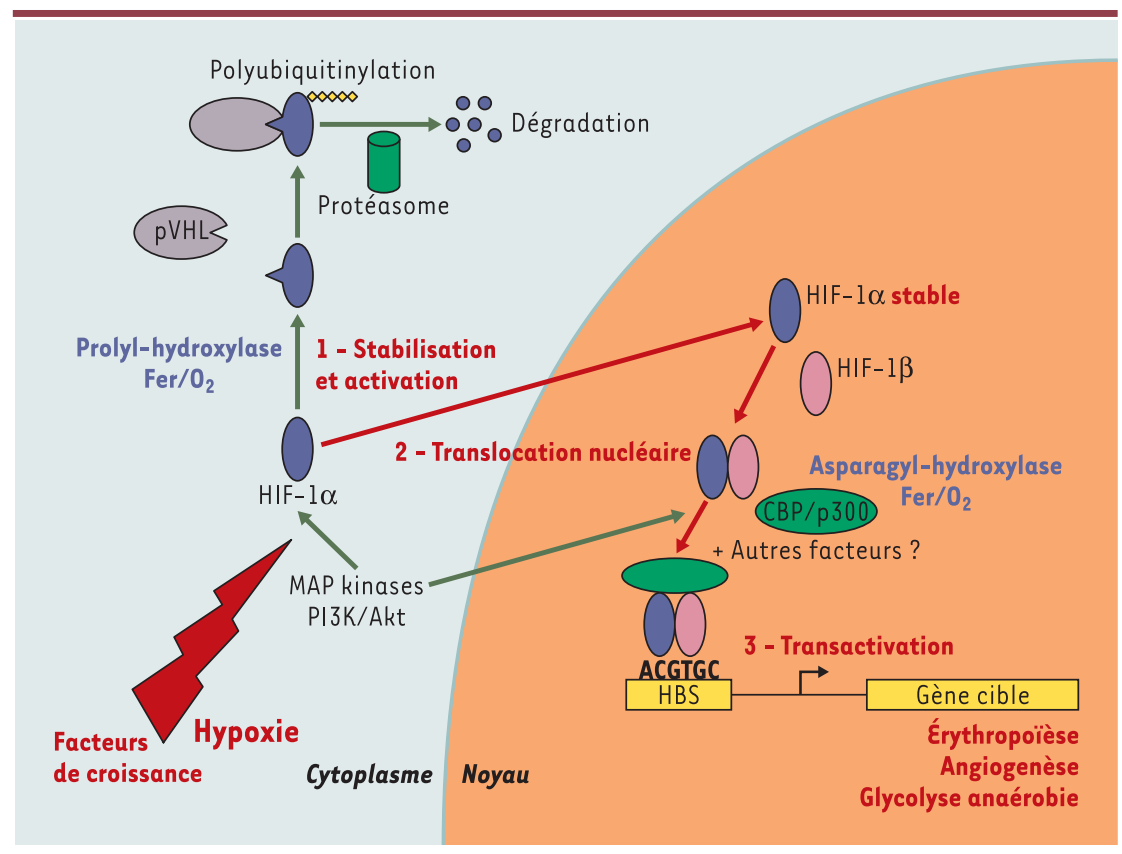

Figure 1. Régulation de l'activité de la protéine HIF-1 $\alpha$ par la concentration intracellulaire en oxygène. La protéine HIF-l $\alpha$ est très instable en présence d'oxygène : elle est rapidement dégradée et sa demi-vie est inférieure à cinq minutes. En effet, une prolyl-hydroxylase hydroxyle un résidu proline de la protéine HIF- $1 \alpha$, ce qui permet son interaction avec la protéine $\mathrm{pVHL}$ (von Hippel Lindau) et sa dégradation par le protéasome. En condition d'hypoxie, la prolylhydroxylase étant inactive, la protéine HIF-l $\alpha$ est très rapidement stabilisée et migre dans le noyau où elle s'associe à son partenaire HIF-1 $\beta$. Le complexe ainsi formé recrute d'autres partenaires, en particulier $\mathrm{CBP} / \mathrm{p} 300$, et se fixe sur la séquence nucléotidique spécifique de reconnaissance du facteur HIF-1 (motif HBS : HIF-binding site) située dans les régions régulatrices des gènes cibles. L'activation de la protéine HIF-1 $\beta$ par l'hypoxie entraîne la synthèse de facteurs protéiques impliqués notamment dans l'érythropoièse, l'angiogenèse et la glycolyse anaérobie. Le recrutement de CBP/p300 par HIF-1 $\alpha$ dans le noyau est également régulé par la tension en oxygène : une asparagyl-hydroxylase va modifier la protéine HIF-l $\alpha$ en présence d'oxygène et empêcher son interaction avec CBP/p300. Notons que de nombreuses autres modifications post-traductionnelles interviennent dans la régulation de l'activité de HIF-1 $\alpha$ [26]. Parmi celles-ci, les phosphorylations de HIF-l $\alpha$ et de ses partenaires par les MAP-kinases (mitogen activated protein kinase) et la PI3K (phosphatidylinositol 3-kinase)/Akt sont impliquées dans l'activation de la voie HIF par de nombreux facteurs de croissance, même en 

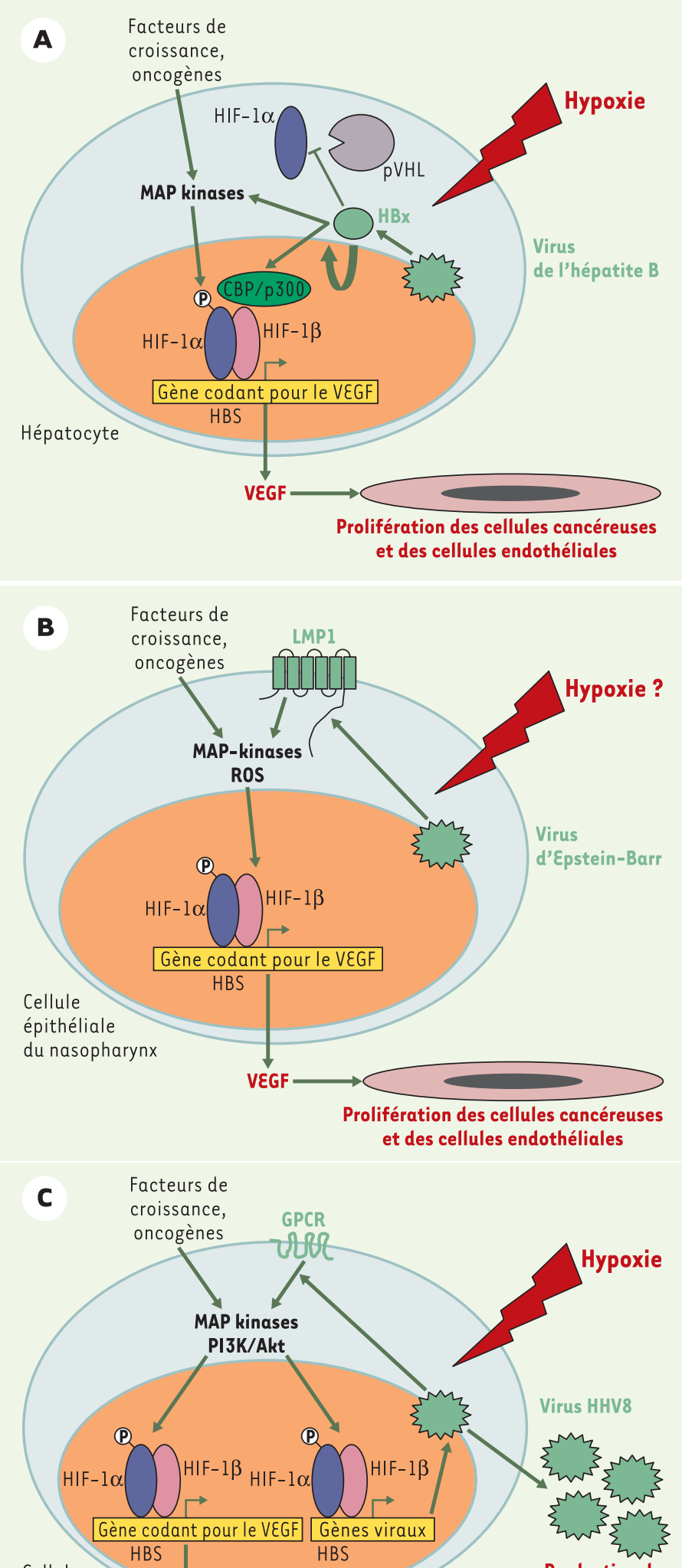

Cellule endothéliale

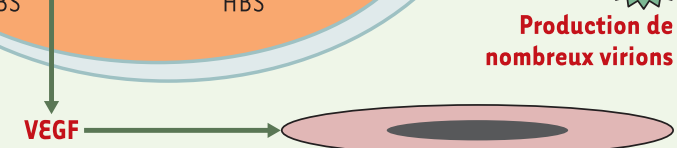

Prolifération des cellules fusiformes et des cellules endothéliales

la protéine virale vGPCR s'associe aux protéines $G$ sousmembranaires et active de manière constitutive les kinases intracellulaires, telles que les MAPK p44/p42 et p38, la PI3K/Akt, ainsi que la synthèse de nombreux facteurs de croissance et cytokines [15]. Parmi ceux$\mathrm{ci}$, on peut citer le VEGF qui permet la prolifération des cellules fusiformes et des cellules endothéliales (Figure 2C). L'activation des kinases cellulaires conduit également à la stabilisation et à la migration nucléaire du

$(\rightarrow) \mathrm{m} / \mathrm{s}$ $2002, n^{\circ} 1$, facteur HIF-l $\alpha(\rightarrow)$, ce qui exacerbe p. 70 encore la synthèse de VEGF au niveau des lésions du sarcome de Kaposi, caractérisées par la prolifération de cellules fusiformes et une angiogenèse importante.

\section{HIF- 1 et expression des gènes viraux}

Le virus HHV8, outre le fait de perturber l'expression d'HIF, est capable d'utiliser ce facteur de transcription pour optimiser sa réplication. En effet, le génome de ce virus possède de nombreux sites de fixation pour le complexe HIF-1 [16]. En condition d'hypoxie, la fixation de HIF-l en amont des gènes viraux stimule l'expression des protéines lytiques et structurales du HHV8 ainsi que sa réplication in vitro dans des cellules de lymphome $B$ des séreuses infectées chroniquement (Figure 2C et Tableau I) [17]. Le sarcome de Kaposi se développe plus particulièrement au niveau de la micro-

Figure 2. Perturbation de la régulation de la protéine HIF-1 $\alpha$ par des protéines virales oncogènes. $A$. La protéine oncogène HBx produite par le virus de l'hépatite B favorise la stabilisation et l'activation de la protéine HIF-l $\alpha$ en inhibant son interaction avec $\mathrm{pVHL}$, en favorisant son interaction avec $\mathrm{CBP} / \mathrm{p300}$ [11] et en activant la voie des MAP-kinases [9, 10]. Le VEGF alors produit stimule la prolifération des cellules cancéreuses et des cellules endothéliales. B. La protéine oncogène membranaire LMPI produite par le virus d'EpsteinBarr stimule les voies des MAP-kinases et des dérivés réactifs de l'oxygène (ROS), entraînant la stabilisation du facteur HIF$1 \alpha$ et la production de VEGF impliqué dans la prolifération des cellules endothéliales et des cellules épithéliales cancéreuses du nasopharynx [13]. C. La protéine membranaire oncogène couplée aux protéines G, GPCR, produite par le virus HHV8 va activer, en collaboration avec des facteurs de croissance et des oncogènes, la voie des MAP-kinases et de la PI3K/Akt [15]. La protéine HIF-l $\alpha$ ainsi stabilisée va s'associer avec ses partenaires nucléaires et activer la synthèse de VEGF qui va induire la prolifération des cellules tumorales et des cellules endothéliales. Le facteur HIF se fixe également au niveau de sites HBS présents dans les promoteurs viraux et augmente la production de particules virales [16, 17]. 
circulation où l'hématocrite, et donc la concentration en oxygène, est très faible. Cet environnement pourrait favoriser d'une part la réplication virale, ainsi que l'expression de protéine(s) oncogène(s), d'autre part la production de facteurs de croissance impliqués dans la prolifération des cellules endothéliales et tumorales.

Le parvovirus humain B19 semble également capable d'utiliser le facteur HIF-1. Ce virus, en infectant et en détruisant les précurseurs érythroïdes, est responsable de crises d'érythroblastopénie aiguë chez les patients atteints de drépanocytose, et de mort fœtale in utero par anasarque fœto-placentaire ${ }^{1}$. Des facteurs érythroïdes spécifiques sont probablement mis en jeu, mais ne semblent pas suffisants pour expliquer le tropisme étroit de ce virus particulièrement difficile à cultiver in vitro [18]. Seule l'infection de cellules humaines de la lignée érythroïde permet une réplication, cependant très limitée, du parvovirus B19. Au laboratoire, nous avons pu montrer que la culture de progéniteurs érythroïdes en hypoxie augmente la synthèse et la production des protéines structurales et non structurales du parvovirus B19 [19]. Cette expression virale accrue en situation d'hy-

${ }^{1}$ CEdème généralisé et intense de l'œuf tout entier (placenta et fœtus), avec production d'ascite. poxie s'explique au moins en partie par la fixation du complexe HIF-1 sur un site HBS présent dans le promoteur viral. L'activation de la réplication du parvovirus B19 en hypoxie s'accompagne d'une augmentation significative de la production de particules virales infectieuses (Tableau l). Des études réalisées in vitro et la mesure de la $\mathrm{pO}_{2}$ intramédullaire montrent que la maturation et la différenciation des précurseurs érythroïdes, cibles du parvovirus B19, s'effectuent à faible concentration d'oxygène $[5,20]$. L'environnement intramédullaire pauvre en $\mathrm{O}_{2}$ et riche en progéniteurs érythroïdes serait favorable à la réplication du parvovirus B19 et à la production de nombreux virions ( $10^{12}$ particules virales/ml de sérum). L'érythropoïèse inefficace et les complications thrombo-emboliques de la drépanocytose participent à l'hypoxie tissulaire et favorisent probablement la réplication du virus chez ces patients. De même, le développement HIF-dépendant $\rightarrow) \mathrm{m} / \mathrm{s}$ 2003, $n^{\circ} 11$, p. 1111 des tissus fœtaux [3] $(\rightarrow)$ pourrait participer à la sensibilité particulière du fœtus à l'infection par le parvovirus B19 (Figure 3).

Notons que des sites HBS sont également retrouvés dans le génome de parvovirus animaux, en particulier le par-

\begin{tabular}{|c|c|c|c|c|c|c|}
\hline & & $\begin{array}{l}\text { Parvovirus } \\
\text { B19 }\end{array}$ & HIV-1 & HHV8 & HBV & $\varepsilon B V$ \\
\hline \multirow{4}{*}{$\begin{array}{l}\text { Impacts de } \\
\text { I'hypoxie }\end{array}$} & Synthèse des protéines virales de structure & $\uparrow(4)$ & $\uparrow(5)$ & $\uparrow(12$ à 22) & ND & ND \\
\hline & Synthèse des protéines virales de régulation & $\uparrow(4)$ & ND & ND & $\uparrow(\mathrm{N} \rho)$ & ND \\
\hline & $\begin{array}{l}\text { Réplication du génome viral et synthèse } \\
\text { de nouveaux virions }\end{array}$ & $\uparrow(5)$ & ND & $\uparrow(>2)$ & ND & ND \\
\hline & Transcription des gènes viraux & $\uparrow(7)$ & $\uparrow(15)$ & ND & $\uparrow(2)$ & ND \\
\hline \multicolumn{2}{|c|}{ Présence de site(s) de fixation pour HIF-1 } & oui & ND & oui? & oui & ND \\
\hline \multicolumn{2}{|c|}{ Autres facteurs de transcription impliqués } & $?$ & NFKB & $?$ & ND (AP-1, ATF/CREB ?) & ND \\
\hline \multicolumn{2}{|c|}{ Activation de voies de transduction } & ND & ND & MAP-kinases & MAP-kinases & $\begin{array}{c}\text { MAP-kinases } \\
\text { ROS }\end{array}$ \\
\hline \multicolumn{2}{|c|}{ Cytokines cellulaires et/ou virales synthétisées } & ND & ND & $\begin{array}{c}\text { VEGF } \\
\text { cellulaire } \\
\text { IL-6 virale }\end{array}$ & VEGF cellulaire & VEGF \\
\hline \multicolumn{2}{|c|}{ Activation d'HIF- $1 \alpha$} & ND & ND & oui & oui & oui \\
\hline
\end{tabular}

Tableau I. Réplication et expression des virus en hypoxie. L'activation de certains promoteurs viraux met en jeu la voie de réponse à l'hypoxie assurée par HIF-1 $\alpha$. La présence et la fonctionnalité de sites de fixation pour HIF-1 $\alpha$ ont été démontrées dans le génome du parvovirus B19 [19] et du virus herpès humain $8[16,17]$. L'activation d'HIF- $1 \alpha$ est impliquée dans le pouvoir pathogène, en particulier oncogène, des virus herpès humains 8 (HHV8), de l'hépatite B (VHB) et d'Epstein-Barr (EBV) [9-15]. La valeur notée entre parenthèses est le rapport entre les données expérimentales obtenues en conditions d'hypoxie (faibles concentrations d'oxygène) et en conditions habituelles de culture cellulaire (20\% d'oxygène). Les cytokines de l'hôte ou les virokines produites en réponse à l'infection virale sont également présentées dans ce tableau. ND : non déterminé. $N \rho$ : non quantifié. 
vovirus $\mathrm{H}-\mathrm{l}$ et le virus minute de la souris. Ces virus non pathogènes pour l'homme possèdent un oncotropisme et des propriétés oncolytiques bien étayés [21] qui leur confèrent un intérêt pour la mise au point de nouveaux traitements de cancers résistants ou inaccessibles aux thérapies conventionnelles. En l'absence d'étude publiée, nous ne pouvons actuellement que spéculer sur le fait que l'environnement intratumoral, présentant une $\mathrm{pO}_{2}$ très faible [7] et des quantités importantes d'HIF- 1 [22], est probablement favorable à l'expression et la réplication in situ de ces virus.

\section{Perspectives thérapeutiques}

Utilisée dans certaines infections à bactéries anaérobies [23], l'oxygénothérapie, en association à des molécules antivirales, pourrait avoir un intérêt dans le traitement d'infections virales. D'ailleurs, l'oxygène hyperbare a déjà été utilisé comme traitement adjuvant de l'infection par le virus de l'immunodéficience humaine de type 1 (VIH-1) [24]. Les bases moléculaires de ce traitement restent mal explorées, puisqu'une seule étude publiée en 1991 a montré que la culture en hypoxie des cellules lymphoïdes de lignée $T$ chroniquement infectées par le VIH-l induit l'augmentation de la synthèse des ARNm rétroviraux, de la protéine de capside p24 et de la glycoprotéine d'enveloppe gpl60 (Tableau I) [25]. Soulignons cependant que les ganglions constituent des carrefours importants du système immunitaire et des « réservoirs » de cellules cibles pour le VIH, dans lesquels la $\mathrm{pO}_{2}$ est d'environ $4 \%$, ce qui pourrait favoriser la réplication in vivo du VIH. Le développement d'agents susceptibles d'interférer avec la voie d'activation du facteur HIF-l $\alpha$ (activateurs d'hydroxylases, phosphatases...) pourrait également constituer une nouvelle voie de recherche pour le traitement de certaines infections virales et des complications néoplasiques que certains virus engendrent.

\section{Conclusions}

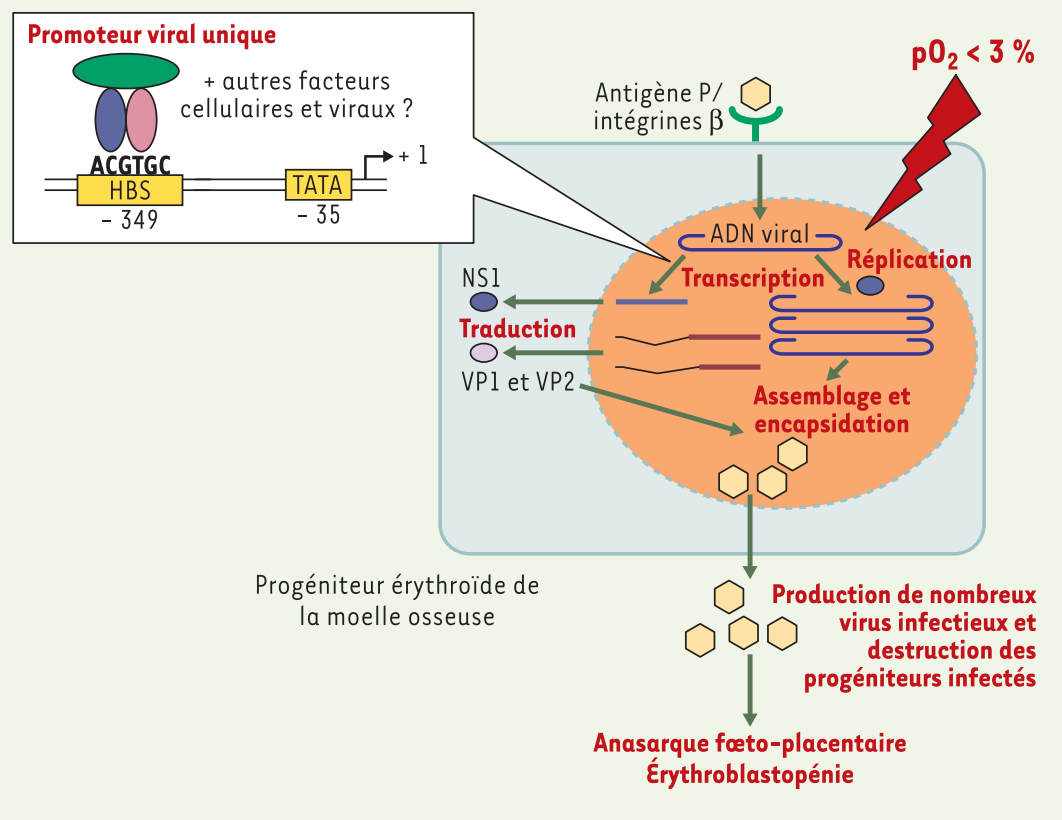

Figure 3. Activation de la réplication et de l'expression du parvovirus B19 par l'hypoxie. Après fixation sur son récepteur, l'antigène $P$ de groupe sanguin, et son corécepteur, un complexe d'intégrines, le parvovirus humain B19 libère son génome dans le noyau des progéniteurs érythroïdes. La transcription et la réplication virales sont activées en condition d'hypoxie in vitro, et probablement in vivo puisque la $\mathrm{pO}_{2}$ a été évaluée à moins de $3 \%$ dans la moelle osseuse. Le promoteur viral qui contrôle l'expression de l'ensemble des gènes viraux possède un site HBS situé à 349 paires de bases en amont du site d'initiation de la transcription [19]. Ce site de fixation pour le facteur HIF-1 $\alpha$, en collaboration probablement avec d'autres sites de fixation pour des facteurs de transcription, est impliqué dans l'augmentation de l'expression du parvovirus B19 in vitro. La stimulation de la production de protéines virales et de virions infectieux en hypoxie pourrait participer à la physiopathologie des érythroblastopénies et des anasarques fœto-placentaires associées à ce virus. NS1, VP1, VP2: protéines virales.
L'interaction des virus avec la voie de réponse à l'hypoxie est encore peu documentée. Cependant, le contrôle de nombreuses fonctions cellulaires par la voie HIF, telles que la régulation du cycle, de l'apoptose et de la différenciation, suggère que d'autres protéines virales sont très certainement susceptibles d'interagir avec ce facteur de transcription. De plus, il nous paraît important de souligner que la culture des virus dans des conditions qui se rapprochent des conditions physiologiques habituelles (type cellulaire, mais aussi $\mathrm{pH}$, concentration en oxygène) permettra la réplication de virus réputés « non cultivables in vitro ». $\Delta$

\section{REMERCIEMENTS}

Les auteurs remercient le Professeur Frédéric Morinet (Virologie et UPR CNRS 9051) pour son soutien scientifique et amical. Les travaux réalisés sur le parvovirus B19 exposés dans cette revue ont été financés par l'Association pour la Recherche sur le Cancer, l'Établissement Français du Sang, l'AP/CNRS et la Fondation pour la Recherche Médicale. 


\section{SUMMARY}

Interaction of viruses with cellular response

to hypoxia

Recent studies show that low oxygen tension levels in cell culture up-regulate the replication of human B19 parvovirus, Kaposi's sarcoma, and human immunodeficiency viruses as well as the expression of viral oncogenic proteins. The mechanisms of this regulation proceed with the major hypoxia-related factor, HIF-l (hypoxia inducible factor-1). HIF-l misregulation is implicated in the oncogenesis potential of some of these viruses. $\diamond$

\section{RÉFÉRENCES}

1. Ebbesen P, Pettersen EO, Denekamp J, et al. Hypoxia, normoxia and hyperoxiaterminology for medical in vitro cell biology. Acta Oncol $2000 ; 39$ : 247-8.

2. Lewis JS, Lee JA, Underwood JC, et al. Macrophage responses to hypoxia : relevance to disease mechanisms. J Leuk Biol 1999 ; 66 : 889-900.

3. Iyer NV, Kotch LE, Agani F, et al. Cellular and developmental control of $\mathrm{O}_{2}$ homeostasis by hypoxia- inducible factor 1 alpha. Genes Dev 1998 ; 12 : 149-62.

4. Schipani $\varepsilon$, Ryan HE, Didrickson S, et al. Hypoxia in cartilage : HIF-lalpha is essential for chondrocyte growth arrest and survival. Genes Dev $2001 ; 15$ : 2865-76.

5. Cipolleschi MG, D'Ippolito G, Bernabei PA, et al. Severe hypoxia enhances the formation of erythroid bursts from human cord blood cells and the maintenance of BFU- $\varepsilon$ in vitro. Exp Hematol 1997 ; 25 : 1187-94.

6. Semenza GL. HIF-1 : mediator of physiological and pathophysiological responses to hypoxia. J Appl Physiol $2000 ; 88$ : 1474-80.

7. Hockel M, Vorndran B, Schlenger K, et al. Tumor oxygenation : a new predictive parameter in locally advanced cancer of the uterine cervix. Gynecol Oncol 1993 ; $51: 141-9$.

8. Hofer T, Wenger H, Gassmann M. Oxygen sensing, HIF-l alpha stabilization and potential therapeutic strategies. Pflug Arch Eur J Physiol 2002 ; 443 : 503-7.

9. Lee SW, Lee YM, Bae SK, et al. Human hepatitis B virus $X$ protein is a possible mediator of hypoxia-induced angiogenesis in hepatocarcinogenesis. Biochem Biophys Res Commun $2000 ; 268: 456-61$.

10. Moon $\varepsilon$ J, Jeong $C H$, Jeong JW, et al. Hepatitis $B$ virus $X$ protein induces angiogenesis by stabilizing hypoxia-inducible factor-1. FASEB J $2004 ; 18: 382-4$.

11. Yoo YG, Cho S, Park S, Lee MO. The carboxy-terminus of the hepatitis $B$ virus $X$ protein is necessary and sufficient for the activation of hypoxia-inducible factor1. FEBS Lett $2004 ; 577: 121-6$.
12. $Y_{0 \circ} Y G, O h S H$, Park $E S$, et al. Hepatitis $B$ virus $X$ protein enhances transcriptional activity of hypoxia-inducible factor-lalpha through activation of mitogenactivated protein kinase pathway. J Biol Chem $2003 ; 278$ : 39076-84.

13. Wakisaka N, Kondo S, Yoshizaki T, et al. Epstein-Barr virus latent membrane protein 1 induces synthesis of hypoxia-inducible factor 1 . Mol Cell Biol $2004 ; 24: 5223-34$.

14. Hui EP, Chan ATC, Pezzella F, et al. Coexpression of hypoxia-inducible factor 1 and 2, carbonic anhydrase IX, and vascular endothelial growth factor in nasopharyngeal carcinoma and relationship to survival. Clin Cancer Res $2002 ; 8$ : 2595-604.

15. Sodhi A, Montaner S, Patel V, et al. The Kaposi's sarcoma-associated herpes virus G protein-coupled receptor up-regulates vascular endothelial growth factor expression and secretion through mitogen-activated protein kinase and p38 pathways acting on hypoxia-inducible factor lalpha. Cancer Res 2000 ; $60: 4873-80$.

16. Haque M, Davis DA, Wang V, et al. Kaposi's sarcoma associated herpesvirus (human herpesvirus 8 ) contains hypoxia response elements : relevance to lytic induction by hypoxia. J Virol $2003 ; 77: 6761-8$.

17. Davis DA, Rinderknecht AS, Zoeteweij JP, et al. Hypoxia induces lytic replication of Kaposi sarcoma-associated herpesvirus. Blood 2001 ; 97 : 3244-50.

18. Pillet S, Morinet F. Parvovirus B19 and erythroid cells. Pathol Biol (Paris) $2002 ; 50$ : 349-56.

19. Pillet S, Le Guyader N, Hofer T, et al. Hypoxia enhances human B19 erythrovirus gene expression in primary erythroid cells. Virology $2004 ; 327: 1-7$.

20. Harrison JS, Rameshwar P, Chang V, Bandari P. Oxygen saturation in the bone marrow of healthy volunteers. Blood $2002 ; 99: 394$.

21. Deleu L, Daeffler L, Faisst S, Rommelaere J. Action oncolytique des parvovirus de rongeurs. Virologie $2002 ; 6: 29-40$.

22. Zhong H, De Marzo AM, Laughner $\varepsilon$, et al. Overexpression of hypoxia-inducible factor lalpha in common human cancers and their metastases. Cancer Res 1999 ; $59: 5830-5$

23. Sugihara $A$, Watanabe $H$, Oohashi $M$, et al. The effect of hyperbaric oxygen therapy on the bout of treatment for soft tissue infections.J Infect $2004 ; 48: 330-3$.

24. Reillo MR, Altieri RJ. HIV antiviral effects of hyperbaric oxygen therapy.J Assoc Nurses AIDS Care $1996 ; 7: 43-5$.

25. Polonis VR, Anderson GR, Vahey MT, et al. Anoxia induces human immunodeficiency virus expression in infected T cell lines. J Biol Chem 1991; 266 : 11421-4.

26. Brahimi-Horn C, Mazure N, Pouysségur J. Signalling via the hypoxia-inducible factor1 requires multiple posttranslational modifications. Cell Signal $2005 ; 17: 1-9$.

\section{TIRÉS À PART}

S. Pillet

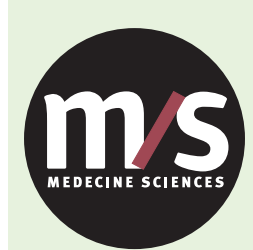

Tarifs d'abonnement M/S - 2005

Abonnez-vous

à Médecine/Sciences
$>$ 1985-2005, depuis 20 ans, grâce à $\mathrm{m} / \mathrm{s}$, vous vivez en direct les progrès des sciences biologiques et médicales

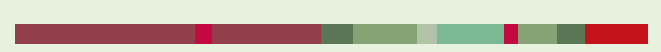

Bulletin d'abonnement page 475 dans ce numéro de $\mathrm{m} / \mathrm{s}$

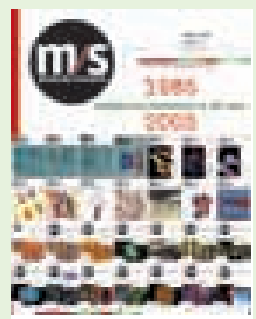

